\title{
Cumplimiento de las recomendaciones de la OMS en noticias sobre suicidios. Análisis de la prensa digital española (2010-2017)
}

\section{Compliance with the WHO guidelines on suicide reporting. An analysis of the Spanish digital press (2010-2017)}
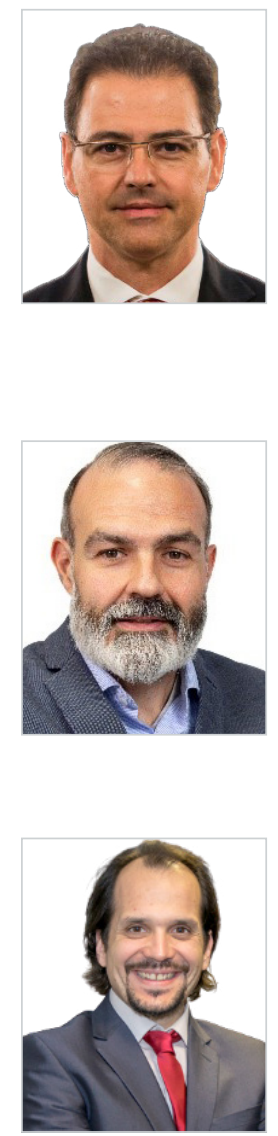

Francisco Javier Olivar de Julián. Doctor en Comunicación (Sociedad del Conocimiento y Acción en los Ámbitos de la Educación, la Comunicación, los Derechos y las Nuevas Tecnologías), Beca de Excelencia UNIR 2017. Máster Universitario en Prevención de Riesgos Laborales. Máster Universitario en Sistemas Integrados de Gestión de la Prevención de Riesgos Laborales, la Calidad, el Medio Ambiente y la Responsabilidad Social Corporativa. Grado en Administración y Dirección de Empresas. Técnico Superior en Prevención de Riesgos Laborales RD 39/1997. Diplomado en Ciencias Empresariales. Miembro del Grupo de investigación TRES-i: Trabajo líquido y riesgos emergentes en la sociedad de la información. Profesor del Máster Universitario en Prevención de Riesgos Laborales y del Máster Universitario en Sistemas Integrados de Gestión de la Prevención de Riesgos Laborales.

Universidad Internacional de la Rioja, España

franciscojavier.olivar@unir.net

ORCID: 0000-0002-2030-2458

Jesús Díaz-Campo. Licenciado y Doctor en Periodismo por la Universidad Complutense de Madrid (UCM). Actualmente es Director de Investigación y Profesor Titular en la Universidad Internacional de la Rioja (UNIR). Está acreditado por ANECA a Profesor Titular de Universidad. Un Sexenio de Investigación (CNEAI). Ha publicado alrededor de 40 artículos en revistas JCR y SCOPUS. Director del grupo de investigación "Comunicación y Sociedad Digital" (COYSODI). Investigador principal del proyecto NewsSharing: Consumo de noticias en medios sociales. Análisis de factores en la selección y difusión de contenidos mediáticos (financiado en la convocatoria de Proyecto de I+D 2017 Retos)

Universidad Internacional de la Rioja, España

jesus.diaz@unir.net

ORCID: 0000-0001-5014-8749

Francisco Segado-Boj. Profesor Ayudante Doctor (Universidad Complutense de Madrid). Doctor en Comunicación (Universidad Complutense de Madrid). Codirector del Grupo de Investigación "Historia y Estructura de la Comunicación y el Entretenimiento", Departamento de Periodismo y Comunicación Global - Facultad de CC. de la Información de la Universidad Complutense de Madrid. Universidad Complutense de Madrid, España fsegado@ucm.es

ORCID: 0000-0001-7750-3755

Cómo citar este artículo:

Olivar de Julián, F. J.; Díaz-Campo, J. y Segado-Boj, F. (2021). Cumplimiento de las recomendaciones de la OMS en noticias sobre suicidios. Análisis de la prensa digital española (2010-2017). Doxa Comunicación, 32, pp. 57-74.

https://doi.org/10.31921/doxacom.n32a3 
Recibido: 10/02/2021 - Aceptado:19/03/2021 - En edición: 16/04/2021 - Publicado: 14/06/2021

\section{Resumen:}

Esta investigación analiza el cumplimiento de las recomendaciones de la Organización Mundial de la Salud (OMS) en las noticias sobre suicidios publicadas en la prensa digital española en el periodo comprendido entre 2010 y 2017. Para ello se ha utilizado la hemeroteca Mynewsonline. Se ha llevado a cabo un análisis de contenido sobre un universo creado a partir de todas las noticias sobre suicidios $(\mathrm{n}=183)$ publicadas en los seis principales medios de comunicación digitales españoles (elpais.com, elmundo.es, abc. es, lavanguardia.com, elconfidencial.com y 20minutos.es). Entre otras cuestiones, se ha estudiado también el acompañamiento gráfico y el carácter joven y/o popular de las víctimas. Los resultados indican que se publican pocas noticias sobre suicidios y que solo el $32,84 \%$ de las publicadas se refieren a sucesos ocurridos en España. También se ha evidenciado una falta del seguimiento de las recomendaciones éticas de la OMS.

Palabras Clave:

OMS; suicidio; prensa digital; noticias; Mynewsonline.
Received: 10/02/2021 - Accepted: 19/03/2021 - Early access: 16/04/2021 - Published: 14/06/2021

\section{Abstract:}

This research analyses compliance with World Health Organization (WHO) guidelines on the reporting of suicides as published in the Spanish digital press over the period between 2010 and 2017. The Mynewsonline newspaper library has been used for this. A content analysis has been carried out on a sample created from all the news reports on suicides $(n=183)$ published in the six main Spanish digital media outlets (elpais.com, elmundo.es, abc.es, lavanguardia. com, elconfidencial.com and20minutos.es). Among other issues, the use of photographs and the young and / or popular character of the victims have also been studied. The results indicate that few news items about suicides are published and that only $32.84 \%$ of those published refer to events that occurred in Spain. A lack of compliance with the ethical recommendations of the WHO is also evident.

\section{Keywords:}

WHO; suicide; digital press; news; Mynewsonline.

\section{Introducción}

\subsection{El suicidio y su repercusión mediática}

Cerca de 800.000 personas mueren en el mundo cada año a causa del suicidio, lo que supone que se produce un fallecimiento por esta causa cada 40 segundos. Por cada muerte se estima que se producen 20 tentativas de suicidio, lo que equivale a decir que cada dos segundos alguna persona intenta suicidarse. Además, ésta es la segunda causa principal de muerte entre las personas que se encuentran entre los 15 y los 29 años de edad (OMS, 2018). En España la primera causa de muerte externa en 2018 fue el suicidio con 3.539 fallecimientos (2.619 hombres y 920 mujeres) (INE, 2018). A la vista de estos datos la muerte auto infligida puede considerarse como un grave problema de salud pública, tanto en España como en el resto del mundo(OMS, 2018; Gobierno de España, 2020).

Estas cifras han intensificado el debate continuo que existe sobre las cifras de fallecimientos por suicidios, que suponen la primera causa de muerte no natural en España (INE, 2018) y el tratamiento informativo que los medios de comunicación ofrecen sobre el suicidio, en donde todavía se mantienen las dudas sobre la conveniencia o no de publicar este tipo de noticias. El miedo a que pueda producirse un efecto imitación (efecto Werther) frente al hecho de que una publicación responsable de este tipo de noticias pueda producir un efecto preventivo (efecto Papageno) sigue siendo todavía objeto de estudio científico. El primer efecto toma su nombre de la novela "Las penas del joven Werther" de Johan Wolfgang Von Goethe (1774) debido a que, justo después de su publicación, se produjo una serie de suicidios de imitación relacionados con el protagonista del libro. Este efecto fue estudiado por el sociólogo David Phillips (1974) quien demostró la relación 
que existía entre las noticias de suicidio publicadas en el New York Times y el número de muertes por dicha causa en Estados Unidos. Investigaciones posteriores han demostrado que determinadas informaciones sobre suicidios no siguen las recomendaciones de la OMS y que este comportamiento poco profesional en la redacción de este tipo de noticias puede contribuir al contagio suicida (Carmichael y Whitley, 2019; Kim et al., 2013).

En contraposición de este efecto se contempla el efecto Papageno (nombre de un hombre-pájaro protagonista de la ópera "La flauta mágica" de Wolfgang Amadeus Mozart, quien es convencido para no suicidarse), que considera que una información sobre suicidios con un enfoque preventivo, en línea con los principios que marca la OMS para la redacción de este tipo de noticias, puede evitar tentativas y suicidios (Durán y Fernández-Beltrán, 2020).

La OMS (2000)propone a los profesionales del periodismo una serie de normas y recomendaciones para "informar del suicidio de manera apropiada, exacta y potencialmente útil a través de medios progresistas e inteligentes puede prevenir una trágica pérdida de vidas":

¿Cómo informar sobre el suicidio en general?:

- Las estadísticas deberán interpretarse cuidadosa y correctamente.

- Deberán usarse fuentes auténticas y confiables.

- Los comentarios espontáneos deberán manejarse con cuidado.

- Las generalizaciones basadas en cifras pequeñas requieren particular atención.

- Se debe oponer resistencia a informar el comportamiento suicida.

¿Cómo informar sobre un suicidio específico?:

- El cubrimiento sensacionalista de suicidios deberá evitarse. Deberá hacerse el mayor esfuerzo por evitar exageraciones. Las fotografías de la víctima, del método empleado y de la escena del suicidio deben evitarse. Los titulares en primera página nunca son la ubicación ideal para informar sobre un suicidio.

- Deberán evitarse las descripciones detalladas del método usado.

- No deberá informarse acerca del suicidio como algo inexplicable o simplista.

- El suicidio no deberá describirse como un método para enfrentar problemas.

- Los informes deberán tener en cuenta el impacto sobre las familias.

- No glorificar a las víctimas de suicidio como mártires y objetos de adulación.

- Describir las consecuencias físicas de intentos de suicidio puede actuar como elemento de disuasión.

Suministro de información sobre ayuda disponible:

- Lista de servicios de salud mental y líneas telefónicas de ayuda disponibles.

- Publicitar las señales de advertencia del comportamiento suicida.

- Ofrecer un mensaje de solidaridad a los sobrevivientes (2000: 9). 
También propone la OMS (2000)unas acciones y omisiones específicas en relación con el suicidio:

Qué hacer:

- Trabajar estrechamente con autoridades de la salud en la presentación de los hechos.

- Referirse al suicidio como un hecho logrado, no uno exitoso.

- Presentar sólo datos relevantes en las páginas interiores.

- Resaltar las alternativas al suicidio.

- Proporcionar información sobre líneas de ayuda y recursos comunitarios.

- Publicitar indicadores de riesgo y señales de advertencia.

Qué no hacer:

- No publicar fotografías o notas suicidas.

- No informar detalles específicos del método usado.

- No dar razones simplistas.

- No glorificar ni sensacionalizar el suicidio.

- No usar estereotipos religiosos o culturales.

- No aportar culpas (2000: 11).

\subsection{Estudios previos sobre suicidios}

El tratamiento del suicidio en los medios de comunicación ha sido objeto de estudio en diferentes investigaciones. En algunos casos el análisis se ha centrado en la cobertura mediática de este tipo de siniestros cuando la víctima corresponde con un personaje famoso (Fink, Santaella-Tenorio y Keyes, 2018; Choi y Oh, 2016; Kim et al., 2013; Müller, 2011) o cuando, independientemente de su grado de popularidad, el estilo de redacción utilizado en la noticia transmite a la audiencia una irreal sensación de control por parte de la víctima, situación que contribuye a estigmatizar todavía más a todo el entorno relacionado con el suicidio (Boudry, 2008). Otros estudios han demostrado que las noticias de suicidios con víctimas famosas tienen una mayor probabilidad de provocar un efecto imitación en la sociedad que este mismo tipo de noticias con víctimas anónimas (Stack, 2005).

También se han hallado estudios con un carácter más orientado a la ética profesional donde se ha analizado la forma en la que se redactan las noticias sobre suicidios. En estas investigaciones se concluye que los medios de comunicación no siempre cumplen las recomendaciones indicadas por la Organización Mundial de la Salud (OMS) (Díaz-Campo et al., 2021; Duncan y Luce, 2020; Camacho Markina y Santos Díez, 2020; Durán y Fernández-Beltrán, 2020; Acosta et al., 2019; Garrido-Fabián et al., 2018; Herrera Ramírez et al., 2015) y que, aunque se comprende el significado de estas directrices, saber interpretar estas recomendaciones no siempre es sencillo (Machlin, Skehan y Sweet, 2012).En relación con la concepción del valor periodístico y la evolución del enfoque ético en las noticias sobre suicidios, se puede decir que se ha pa- 
sado de un género popular de historias sensacionales a un asunto de carácter privado que necesita prominencia, impacto o rareza para ser publicado (Parks, 2019).

Otros estudios cualitativos señalan que el tratamiento periodístico sobre el suicidio es diferente al de otras muertes no naturales y que, aunque inicialmente la línea que siguen los medios está mayormente orientada a no publicar este tipo de noticias, los periodistas finalmente acaban aceptando su redacción a través de un proceso personal de racionalización (Beam, John y Yaqub, 2017). En relación con otros tipos de muerte no natural, como por ejemplo los homicidios, se han realizado estudios que indican una mayor presencia en los medios de estos últimos que en el caso de los suicidios, circunstancia que contribuye a minimizar los recursos disponibles para la prevención del suicidio (Niederkrotenthaler y Stack, 2017).

En este sentido, se han publicado recientemente algunos artículos científicos que animan a romper el silencio de los medios de comunicación y a redactar con mayor profesionalidad y con un enfoque preventivo este tipo de noticias (Till, Tran y Niederkrotenthaler, 2020; Yaqub, Beam y John, 2020) y las que tienen que ver con violencia en general (Díaz-Campo, Chaparro-Domínguez y Rodríguez-Martínez, 2018) o con los incendios (Pérez Pereiro, Chaparro Dominguez y Díaz del Campo, 2018).

Asímismo, existen estudios en los que se anima a los gobiernos y a las organizaciones de la salud a que se involucren en la difusión de guías especializadas para los profesionales del periodismo de sucesos (Gandy y Terrion, 2015).

Otros trabajos analizan el impacto del uso de las redes sociales en la actitud hacia el suicidio(Choi y Noh, 2020) y la utilización de campañas preventivas específicas para reducir la mortalidad por esta causa (Sindoni, 2020; Pirkis et al., 2019). Incluso los estudios más actuales contemplan la incidencia de la COVID 19 en relación con el suicidio (Wake, Paton y Pryor, 2020).

En relación con investigaciones de carácter multivariable sobre noticias de suicidios existe una investigación que identifica métodos, fuentes y grado de adherencia a las recomendaciones de la OMS (Victor et al., 2019), pero no se han encontrado estudios que analicen el cumplimiento de las recomendaciones de la OMS junto al acompañamiento gráfico y a las víctimas jóvenes y/o famosas.

Por tanto, la novedad de este estudio radica principalmente en este análisis multivariable en relación con el cumplimiento de las recomendaciones de la OMS en la redacción de noticias sobre suicidios en los medios de comunicación españoles.

\subsection{Objetivos}

Objetivo general:

1.3.1. Identificar el cumplimiento o incumplimiento de las recomendaciones de la OMS para los profesionales de los medios de comunicación en la redacción de noticias sobre suicidios publicadas por la prensa digital española.

Objeticos específicos:

1.3.2. Medir la presencia de noticias sobre suicidios en la prensa digital española en el periodo 2010-2017.

1.3.3. Identificar la relación delos diferentes tipos de incumplimientos con el acompañamiento gráfico. 
1.3.4. Identificar la relación de los diferentes tipos de incumplimientos con el tipo de víctima (joven, no joven, famoso o desconocido).

\section{Metodología}

En este estudio se ha realizado un análisis de contenido cuantitativo del tratamiento informativo que ofrecen los medios digitales españoles sobre las noticias de suicidios.

\subsection{Determinación de la muestra}

La unidad de análisis elegida para este estudio ha sido la pieza periodística relacionada con el suicidio, publicada por los diarios digitales seleccionados durante el periodo 2010-2017.

Para este estudio se han seleccionado únicamente medios digitales debido a la creciente tendencia de los lectores a informarse más en internet y menos en la prensa de papel (Boasberg et al, 2019) (ver Figura 1).

Figura 1. Evolución de la audiencia de diarios según tipo de lector (papel/internet), 2000-2018

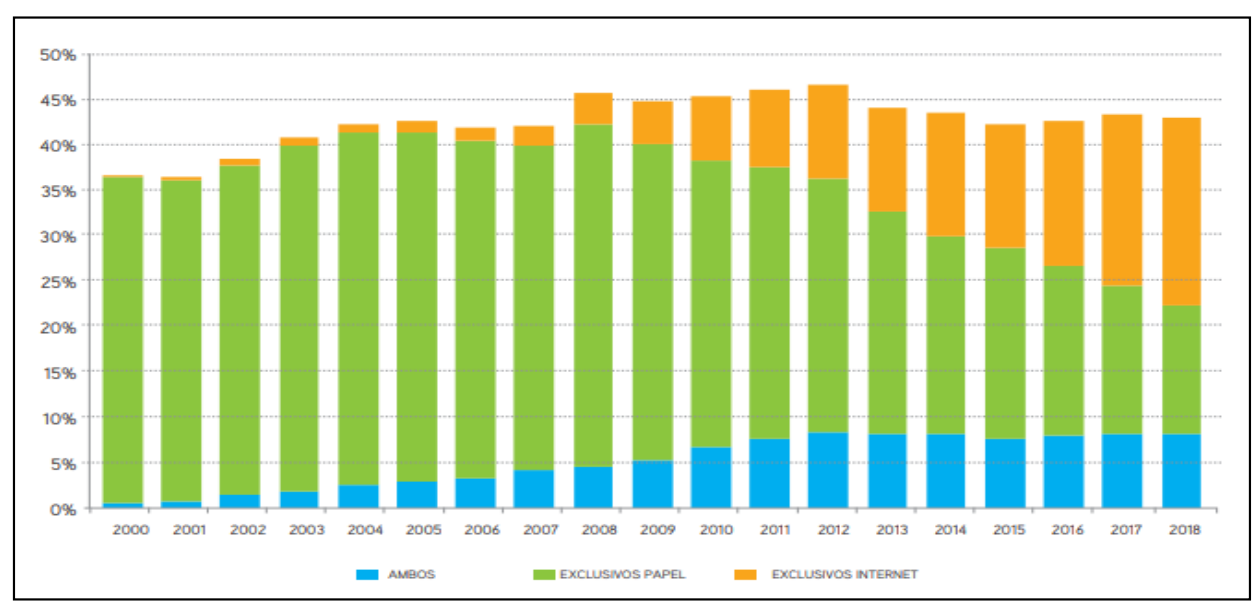

Fuente: Asociación para la investigación de medios de comunicación (AIMC), Marco general de los medios en España, (Boasberget al, 2019)

Para obtener una muestra representativa de los diarios online se han tenido en cuenta tanto a diarios inmigrantes como nativos digitales. Los diarios inmigrantes digitales son los que han realizado una "adaptación de los periódicos tradicionales a los nuevos medios digitales y su interfaz” (Peña-Fernández, Lazkano-Arrillaga, y García-González, 2016: 27). Por otro lado, se han considerado diarios nativos digitales a todos aquellos que han nacido como diarios online, teniendo también en cuenta a los que se han sufrido una rápida transformación digital, como por ejemplo 20minutos.es, que pasa de ser periódico tradicional a digital en tan solo cinco años (2000-2005), disponiendo de licencia Creative Commons (que 
permite la copia literal de sus textos citando la fuente) y ofreciendo una total apertura de sus contenidos a los comentarios de los lectores (López Redondo, 2012).

Para elegir a los medios de comunicación, se han adoptado criterios de predominancia y difusión según los índices de audiencia (número de visitas/mes y número de usuarios únicos). En base a ello, se han seleccionado los diarios inmigrantes digitales, elpais.com, elmundo.es, abc.es y lavanguardia.com y los diarios nativos digitales elconfidencial.com y 20minutos.es.

Los datos de audiencia han sido extraídos del Estudio General de Medios (Boasberg, 2019) y de la empresa de medición de audiencias en internet ComScore (2017), entidades habituales de consulta para este tipo de estudios tanto nacionales (Galletero Campos y Saiz Echezarreta, 2018; Rodríguez, 2018), como internacionales (Potvin Kent y Pauzé, 2018) and the healthfulness of all food and beverage ads was assessed using the Pan-American Health Organization (PAHO).

Para la selección de noticias se han creado unos criterios de inclusión/exclusión en base a las recomendaciones tomadas para otros estudios científicos anteriores, como por ejemplo el de Zimmermann et al (2019).

Se han seleccionado noticias de suicidios en España y en cualquier lugar del mundo durante el periodo 2010-2017, contemplando tanto tentativas como hechos consumados, publicadas en los medios de comunicación seleccionados. Se han aceptado las noticias con carácter de suceso actual, dejando fuera del estudio las recopilaciones o resúmenes de noticias, reportajes temáticos, comentarios de opinión, reivindicaciones o balances estadísticos.

\subsubsection{Código de análisis y variables}

Primeramente, se realizó una fase de prueba y un pre-test con el objetivo de valorar la conveniencia y eficacia de las variables. Una vez determinadas las variables óptimas para el estudio se creó el cuadro de categorías que se detalla a continuación (ver Tabla 1). 
Cumplimiento de las recomendaciones de la OMS en noticias sobre suicidios. Análisis de la prensa digital...

Tabla 1. Cuadro de variables con detalle según bloques de categorías

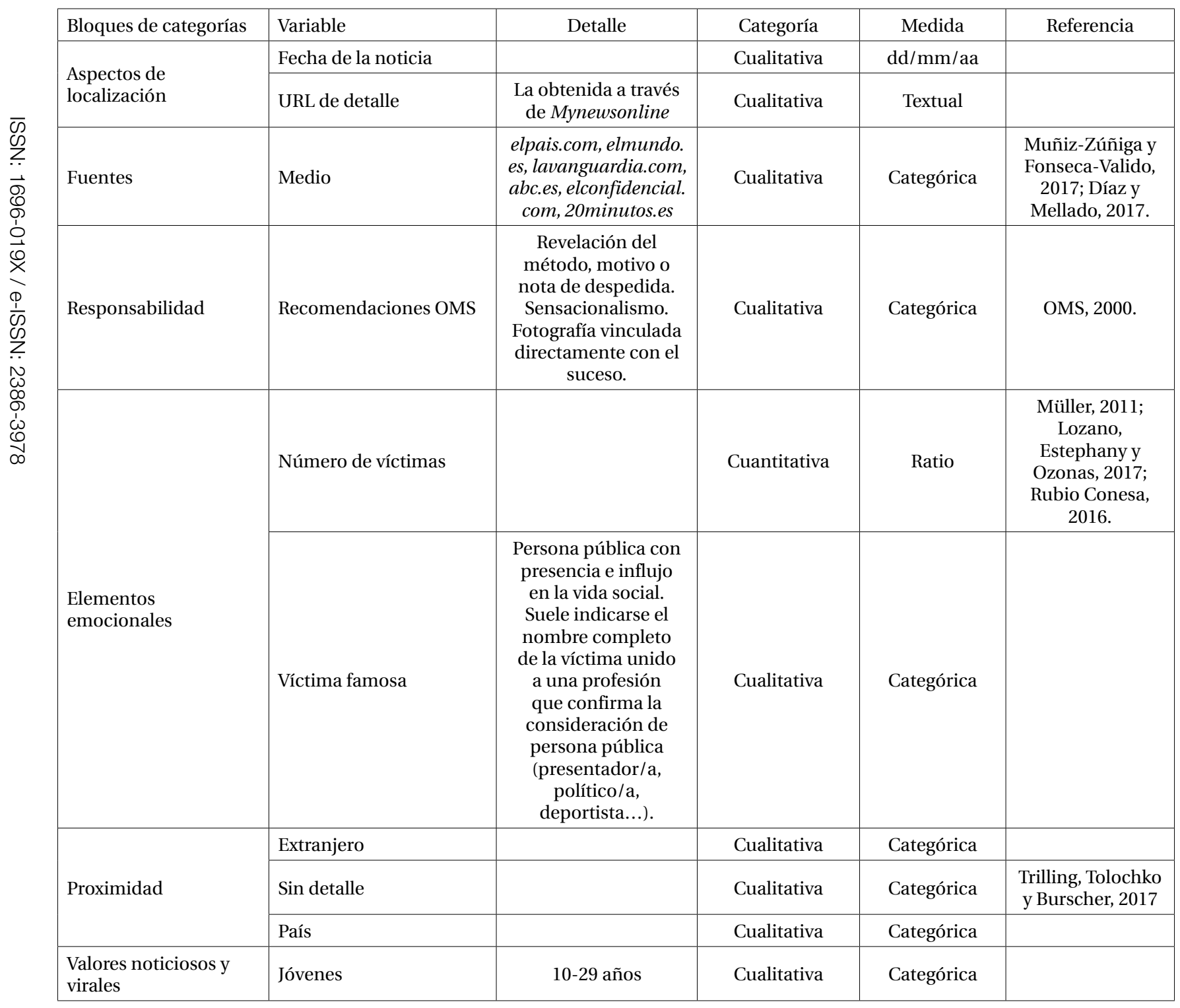

Fuente: elaboración propia 
Para determinar objetivamente los parámetros que identifican la variable "víctima joven", al igual que en otros estudios (Olivar-Julián, y Díaz-Campo, 2020), se ha determinado la franja de edad entre 10 y 29 años (ambos incluidos), que queda dentro de los rangos de consulta disponibles en el INE. Esta decisión se alinea con los parámetros que marcan otras entidades como la Organización de Naciones Unidas (ONU) (10-24 años), la OMS (10-30 años), el Centro de Investigaciones Sociológicas (CIS) (15-29 años) y el Instituto de la Juventud de España (INJUVE) (15-29 años)(RTVE, 2019).

\subsubsection{Muestra}

La muestra se ha obtenido de la hemeroteca digital Mynewsonline, que dispone de publicaciones de prensa online desde 2010. Este recurso se ha utilizado también en otras investigaciones similares relacionadas con la comunicación (Repiso y Chaparro-Domínguez, 2018; Garcia-Gil y Cortiñas-Rovira, 2018).

En esta hemeroteca digital se ha procedido a realizar una búsqueda avanzada de noticias de suicidios según los criterios de inclusión/exclusión indicados anteriormente dentro del periodo 2010-2017. Esta búsqueda ha dado como resultado la localización de183 noticias publicadas en los medios de comunicación seleccionados.

\section{Resultados}

\subsection{Cumplimiento de las recomendaciones de la OMS}

Se ha detectado la existencia algunos incumplimientos sobre las recomendaciones que la OMS ofrece a los profesionales de los medios de comunicación que elaboran noticias sobre suicidios. Las principales faltas sobre estas indicaciones se han debido a la revelación del método utilizado en el suicidio (162), del motivo que dio lugar a dicha acción (133), de la existencia de una nota de despedida -en ocasiones con detalles concretos de la misma- (30) y el acompañamiento de una fotografía íntimamente relacionada con el suceso (15) (ver Tabla 2).

Tabla 2. Cuadro de incumplimientos en la redacción de noticias sobre suicidios

\begin{tabular}{|lr|}
\hline Revelación del método utilizado en el suicidio & 162 \\
\hline Revelación del motivo del suicidio & 133 \\
\hline Información sobre la nota personal de la víctima & 30 \\
\hline Fotografía vinculada al suicidio & 35 \\
\hline
\end{tabular}

Fuente: elaboración propia

\subsection{Presencia de noticias sobre suicidios en la prensa española}

Se han codificado un total de 183 noticias sobre suicidios en los seis medios de comunicación seleccionados para este estudio. El diario que ha publicado un mayor número de noticias sobre suicidios ha sido elmundo.es (51), seguido de $a b c$. es (37), lavanguardia.com (36), elconfidencial.com (24), elpaís.com (21) y 20minutos.es (14). 
Enla Figura 2 se presenta la evolución de las noticias publicadas sobre suicidios en cada uno de los medios de comunicación.

Figura 2. Evolución del número de noticias de suicidios según medio de comunicación 2010-2017

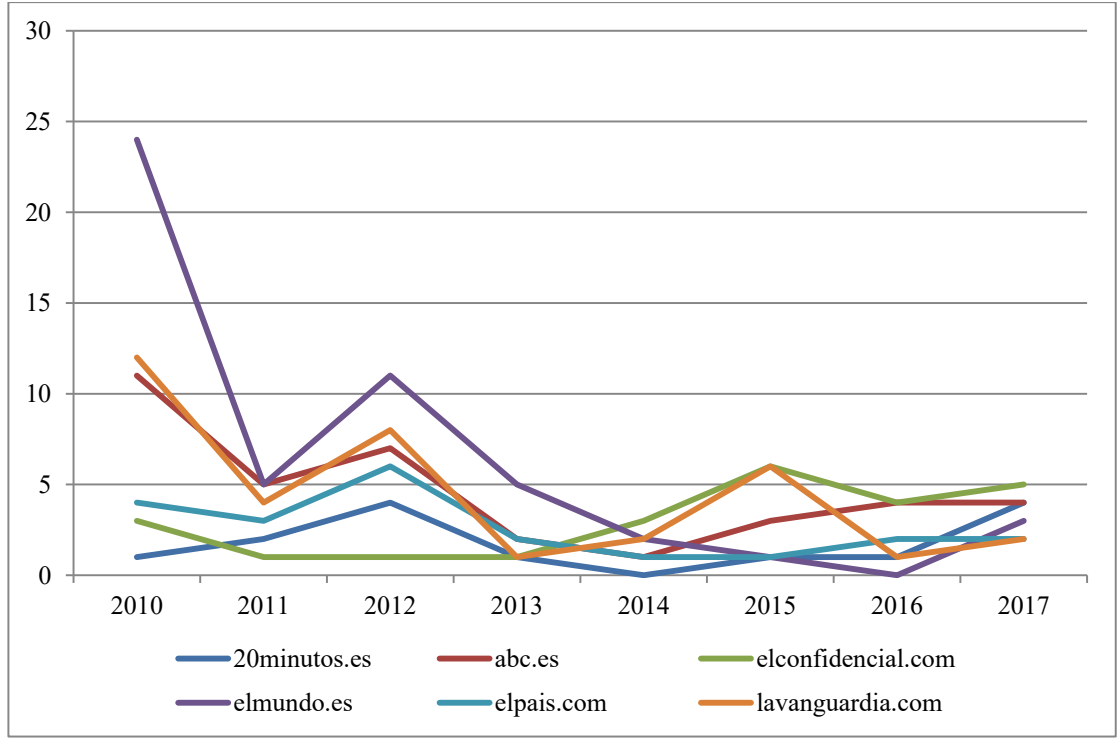

Fuente: elaboración propia

Se ha calculado también la evolución que ha sufrido el número total de noticias sobre suicidios durante el periodo 20102017. En la Figura 3 se observa una tendencia a publicar cada vez un menor número de noticias sobre esta causa de muerte. 
Figura 3. Evolución del número total de noticias de suicidios 2010-2017

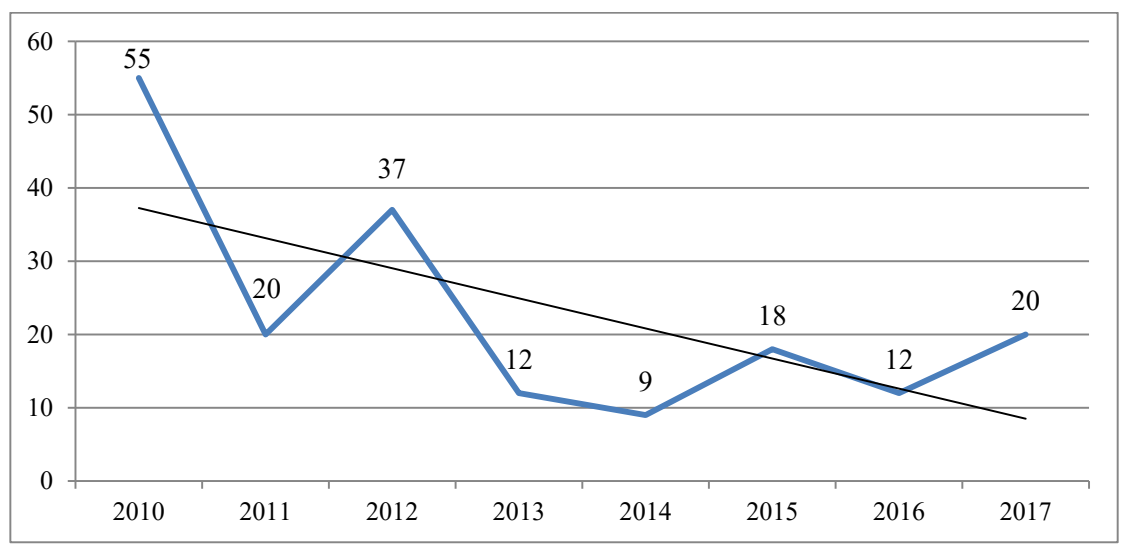

Fuente: elaboración propia

En cuanto al número de noticias de suicidios sucedidos en España (59), se observa que la cifra es sensiblemente inferior a los sucedidos fuera de España (124), pero se aprecia un cambio de tendencia a lo largo del periodo 2010-2017 (ver Figura 4).

Figura 4. Evolución del número total de noticias de suicidios sucedidas en España y fuera de España 2010-2017

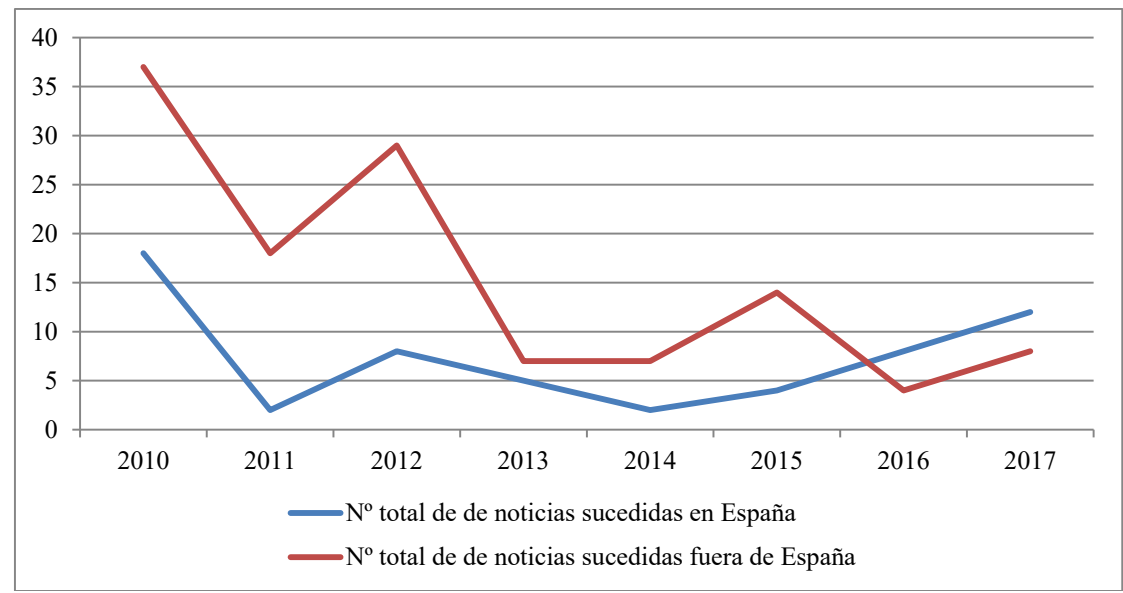

Fuente: elaboración propia 


\subsection{Incumplimientos relacionados con el acompañamiento gráfico}

De un total de 183 noticias estudiadas, 85 venían acompañadas de fotografía $(46,44 \%)$, y solo 15 estaban vinculadas con la noticia $(8,20 \%)$. Los datos recogidos en el capítulo anterior indican que este incumplimiento es el más bajo respecto a los otros tipos contemplados, pero si en este mismo análisis se tienen en cuenta todas las fotografías publicadas (en algunas noticias se publicaba más de una fotografía) el total sumaría 107, de las que 24 (22,43\%) guardan vinculación con el hecho relatado (ver Tabla 3).

Tabla 3. Cuadro de incumplimientos relacionado con el acompañamiento gráfico

\begin{tabular}{|lr|}
\hline No de fotografías totales & 85 \\
\hline No de noticias con fotografía & 107 \\
\hline No de fotografías no vinculadas & 83 \\
\hline No de fotografías vinculadas & 24 \\
\hline
\end{tabular}

Fuente: elaboración propia

Si ponderamos estos datos según el número de noticias publicadas cada año, los datos no difieren mucho del análisis anterior, pero sí se observa un mantenimiento del número de fotografías publicadas hasta $2015 \mathrm{y}$ un claro descenso del número de fotografías no vinculadas en 2017 frente al número total, que es el año que presenta los valores más altos de toda la serie (ver Figura 5).

Figura 5. Evolución ponderada del número de noticias con fotografía y del número de fotografías (vinculadas y no vinculadas con la noticia) $2010-2017$

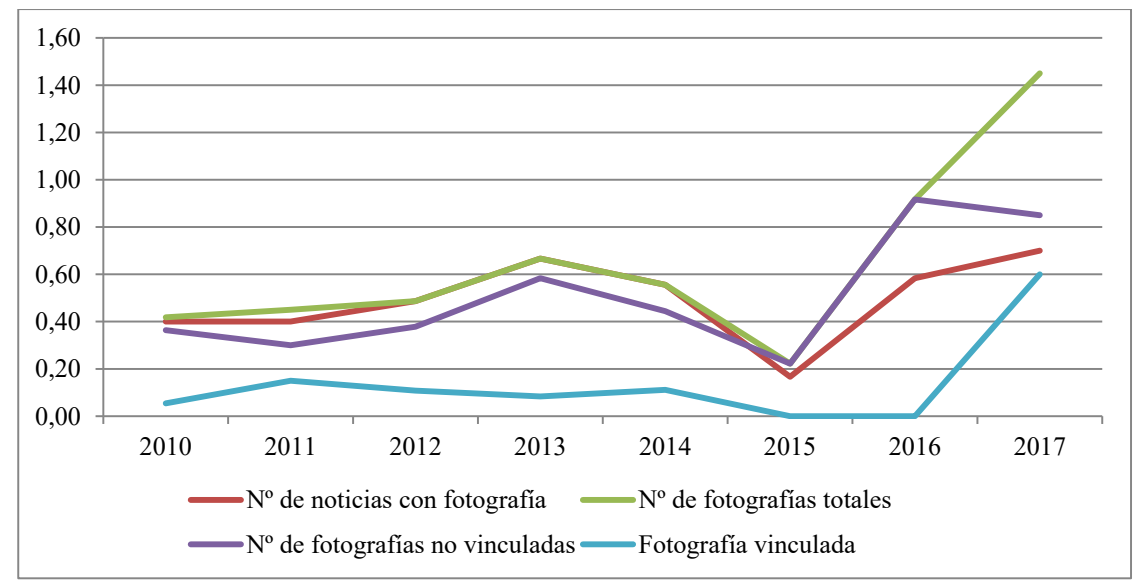

Fuente: elaboración propia 


\subsection{Relación de los incumplimientos con el tipo de víctima (joven, no joven, famoso o no famoso)}

El número de noticias sobre suicidios relacionados con jóvenes en el periodo 2010-2017 es de 58 (31,69\%) mientras el de noticias sobre suicidios con víctimas famosas, el número de publicaciones es de 47 (25,68\%). En el caso de realizar una valoración del número de noticias con ambas variables (víctima joven y famosa) se han detectado 8 noticias que contemplan simultáneamente estas dos categorías (4,37\%). Este escaso porcentaje de coincidencias con ambas variables nos permite poder valorarlas por separado y resaltar el mayor número de publicaciones de noticias sobre suicidios con víctimas jóvenes frente al número de víctimas famosas y destacar que en todas las noticias con víctimas jóvenes se han incumplido las recomendaciones de la OMS, algo que no ocurre en las noticias con víctimas famosas (al menos en los años 2010 y 2016). Podemos comprobar este efecto en la Figura 6.

Figura 6. Evolución ponderada del número de noticias con víctimas jóvenes y víctimas famosas (incluyendo incumplimientos de las recomendaciones de la OMS) 2010-2017

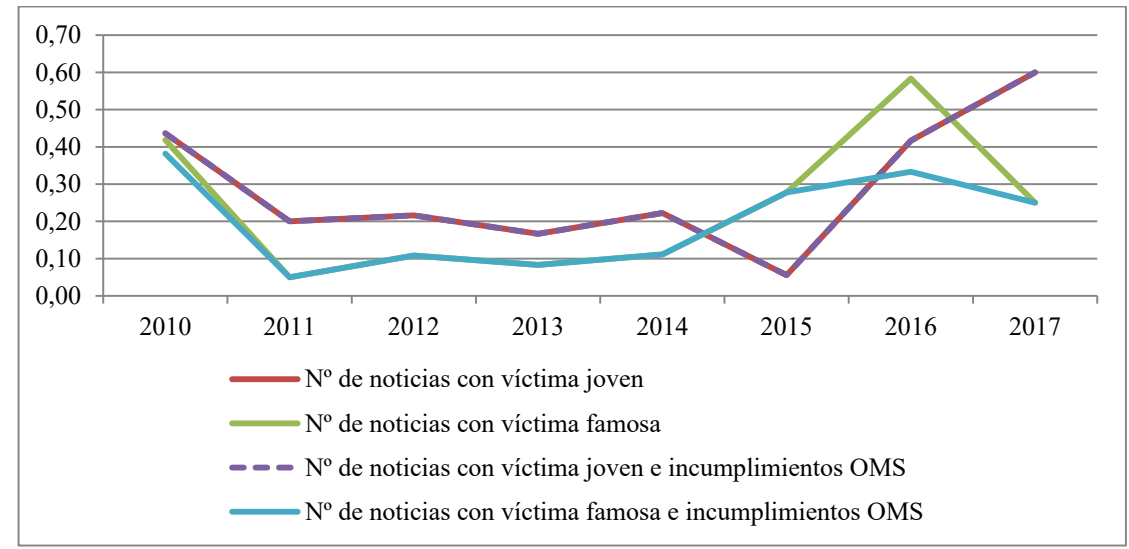

Fuente: elaboración propia

\section{Discusión}

La publicación de noticias sobre suicidios es muy escasa siendo elmundo.es, abc.es y lavanguardia.com los medios que más noticias de este tipo publican. Además, cada vez se tiende a publicar un menor número de noticias sobre esta causa de muerte y solo el $32,24 \%$ de estas noticias se refieren a suicidios sucedidos en España.

Por otro lado, se ha apreciado que los periodistas que redactan este tipo de noticias no atienden a las recomendaciones de la OMS, especialmente revelando el método utilizado en el suicidio y el motivo que dio lugar a dicha acción. 
También se ha observado que las fotografías que acompañan a las noticias de suicidios guardan una vinculación con los hechos relatados en un 22,43\% de los casos y que en 2017 se han publicado de manera ponderada más del doble de fotografías de las que se venían publicando los últimos siete años, siendo estas de un carácter cada vez más vinculante con la noticia.

Un dato llamativo es que todas las noticias con víctimas jóvenes (100\%) incumplen las recomendaciones que la OMS establece para la redacción de noticias sobre suicidios. No ocurre lo mismo en las noticias con víctimas famosas en las que, a pesar de obtener un alto índice de incumplimientos, como también se han detectado en otros estudios similares (Fink, Santaella-Tenorio y Keyes, 2018; Müller, 2011), en alguna ocasión presentan una mayor formalidad en su redacción que en el caso de víctimas jóvenes. Este dato contrasta con los estudios que indican que las noticias de suicidios con víctimas famosas producen un mayor efecto imitación en la sociedad que en el caso de que las víctimas sean anónimas (Stack, 2005).

Por otra parte, e independientemente de las características particulares de la víctima, múltiples estudios han evidenciado que, en general, los medios de comunicación no siguen habitualmente las recomendaciones de la OMS en la redacción de estas noticias (Duncan y Luce, 2020; Camacho Markina y Santos Díez, 2020; Durán y Fernández-Beltrán, 2020; Acosta et al., 2019; Garrido-Fabián et al., 2018;Herrera Ramírez et al., 2015) y muchas otras conclusiones científicas animan a dar voz a este problema social con un enfoque profesional y preventivo (Till, Tran y Niederkrotenthaler, 2020; Yaqub, Beam y John, 2020), tanto en la prensa como en las redes sociales (Choi y Noh, 2020). En todo caso, parece evidente que no siempre es sencillo interpretar estas recomendaciones (Machlin, Skehan y Sweet, 2012) y que sería interesante y recomendable que tanto los gobiernos como las autoridades de la salud pudieran tomar conciencia para crear y divulgar entre los profesionales del periodismo las oportunas guías especializadas que deberían ser consultadas para redactar con una mayor corrección este tipo de noticias tan especiales (Gandy y Terrion, 2015).

Con este planteamiento indicado anteriormente, en el que se publican pocas noticias sobre el suicidio y que, en general, son redactadas para llamar la atención del lector, futuras investigaciones podrían preguntarse por el posible efecto que podría llegar a tener una publicación responsable, con un enfoque más profesional sobre este problema de salud pública. Asimismo, a la vista de los resultados obtenidos, se podría ahondar en la investigar sobre si el hecho de que incumplan las recomendaciones de la OMS en noticias sobre suicidios con víctimas jóvenes pueda llevar implícito que dichas noticias produzcan un mayor efecto imitación en la sociedad que las noticias con víctimas de otros rangos de edad.

En definitiva, a la vista de los resultados, se puede concluir que durante los últimos años los medios digitales de comunicación españoles no han utilizado un tratamiento periodístico especializado en las noticias de suicidios y en general no se ha realizado un serio seguimiento de las recomendaciones de la OMS para la redacción de este tipo de noticias.

\section{Referencias bibliográficas}

Acosta, F. J. et al (2019).«Suicide Coverage in the Digital Press Media: Adherence to World Health Organization Guidelines and Effectiveness of Different Interventions Aimed at Media Professionals», Health Communication, pp. 1-10. doi: 10.1080/10410236.2019.1654176. 
Beam, R.A., John, S.L., Yaqub, M.M. (2017). We don't cover suicide ... (Except when we do cover suicide). Journalism Studies 19: 1447-1465.

Boasberg, J. et al (2019). «Marco general de los medios en España», Duke Law Journal, 1(1), pp. 1-13. doi: 10.1017/ CBO9781107415324.004.

Boudry, V. (2008). Suicide story frames contribute to stigma. Newspaper Research Journal 29(2): 55-69.

Camacho Markina, I. y Santos Díez, M. T. (2020). «El reflejo de las recomendaciones de la OMS para la prevención del suicidio en la prensa española», Estudios sobre el Mensaje Periodístico, 26(3), pp. 903-913. doi: 10.5209/esmp.65239.

Carmichael, V. y Whitley, R. (2019).«Media coverage of Robin Williams' suicide in the United States: A contributor to contagion?», PLOS ONE, 14(5). doi: 10.1371/journal.pone.0216543.

Choi, D.-H. y Noh, G.-Y. (2020).«The influence of social media use on attitude toward suicide through psychological well-being, social isolation, and social support», Information, Communication \& Society, 23(10), pp. 1427-1443. doi: 10.1080/1369118X.2019.1574860.

Choi, Y. J. y Oh, H. (2016).«Does Media Coverage of a Celebrity Suicide Trigger Copycat Suicides?: Evidence from Korean Cases», Journal of Media Economics, 29(2), pp. 92-105. doi: 10.1080/08997764.2016.1170020.

ComScore (2017).Rating \& Planning - Media Rating. Disponible en: https://www.comscore.com/esl/Productos/ Ratings-and-Planning/Media-Ratings.

Díaz, M. y Mellado, C. (2017). «Agenda y uso de fuentes en los titulares y noticias centrales de los medios informativos chilenos. Un estudio de la prensa impresa, online, radio y televisión», Cuadernos.info, 40(40), pp. 107-121. doi: 10.7764/ cdi.40.1106.

Díaz-Campo, J., Chaparro-Domínguez, M. Á y Rodríguez-Martínez, R. (2018). «Los atentados terroristas de Barcelona y Cambrils en la prensa online. Tratamiento informativo en El periódico, El país y The guardian». Profesional de la Información, 27(6), 1358-1367.

Díaz-Campo J, Gómez-García S, Segado-Boj F, Remacha-González L. Ética periodística y Covid-19: análisis de contenido de los códigos deontológicos. Interface (Botucatu). 2021; 25(Supl. 1): e200716

Duncan, S. y Luce, A. (2020).«Using the Responsible Suicide Reporting Model to increase adherence to global media reporting guidelines», Journalism, p. 146488492095268. doi: 10.1177/1464884920952685.

Durán, Á. y Fernández-Beltrán, F. (2020). «Responsabilidad de los medios en la prevención del suicidio. Tratamiento informativo en los medios españoles», El Profesional de la Información, 29(2). doi: 10.3145/epi.2020.mar.07.

Fink, D. S., Santaella-Tenorio, J. y Keyes, K. M. (2018).«Increase in suicides the months after the death of Robin Williams in the US», PLOS ONE, 13(2), p. 191405. doi: 10.1371/journal.pone.0191405.

Galletero Campos, B. y Saiz Echezarreta, V. (2018). «Estudio exploratorio de la calidad en el periodismo digital en Castilla-La Mancha», Barataria. Revista Castellano-Manchega de Ciencias Sociales, 24(24), pp. 173-189. doi: 10.20932/barataria. v0i24.403. 
Gandy, J., Terrion, J. L. (2015). Journalism and suicide reporting guidelines in Canada: Perspectives, partnerships and processes. International Journal of Mental Health Promotion, 17(5), 249-260.

García-Gil, J. y Cortiñas-Rovira, S. (2018).«Quality of recommendations on health-enhancing physical activity in the press. Content analysis of five Spanish newspapers», Apunts. Medicina de l'Esport, 53(199), pp. 113-122. doi: 10.1016/j. apunts.2017.06.002.

Garrido-Fabián, F., Eleazar Serrano-López, A. y Catalán-Matamoros, C. (2018). «El uso por los periodistas de las recomendaciones de la OMS para la prevención del suicidio. El caso del periódico Abc», Revista Latina de Comunicación Social. La Laguna, Tenerife, 73, pp. 810-827. doi: 10.4185/RLCS-2018-1283.

Gobierno de España (2020).Sanidad publica un documento de recomendaciones a los medios de comunicación para las informaciones sobre las conductas suicidas. Disponible en: https: / /www.mscbs.gob.es/gabinete/ notasPrensa.do?id=5006.

Herrera Ramírez, R., Ures Villar, M. B. y Martínez Jambrina, J. J. (2015). «El tratamiento del suicidio en la prensa española: ¿efecto werther o efecto papageno?», Revista de la Asociación Española de Neuropsiquiatría, 35(125), pp. 123-134. doi: 10.4321/S0211-57352015000100009.

INE (2018).Indicadores demográficos básicos. Disponible en: https://www.ine.es/metodologia/t20/metodologia_idb.pdf (Accedido: 2 de junio de 2019).

Kim, J.-H. et al. (2013). «The Werther Effect of Two Celebrity Suicides: an Entertainer and a Politician», PLoS ONE. Editado por A. Mesoudi, 8(12), p. e84876. doi: 10.1371/journal.pone.0084876.

López Redondo, I. (2012).El tratamiento del videojuego: de la prensa generalista a las revistas especializadas. Análisis comparativo de las ediciones impresas y digitales de El Páls, El Mundo, Público y 20 Minutos. Universidad de Sevilla.

Lozano, V., Estephany, A. y Ozonas, C. P. (2017). «Análisis del tratamiento de las noticias de sucesos en los noticiarios matutinos de las televisiones privadas de Ecuador y España», Universitat Autònoma de Barcelona. Facultat de Ciències de la Comunicació.

Machlin, A, Skehan, J, Sweet, M. (2012). Reporting suicide: Interpreting media guidelines. Australian Journalism Review 34(2): 45-56.

Müller, F. (2011). «El “Efecto Werther”: gestión de la información del suicidio por la prensa española en el caso de Antonio Flores y su repercusión en los receptores.», Cuadernos de Gestión de Información, 11(1), pp. 65-7.

Muñiz-Zúñiga, V., \& Fonseca-Valido, R. Á. (2017). «Uso de géneros periodísticos, valores noticia y fuentes de información en los medios de comunicación de Santiago de Cuba», Vivat Academia, 138, pp. 120-140.

Niederkrotenthaler, T, Stack, S. (2017). Media and Suicide: International Perspectives on Research, Theory, and Policy. London: Routledge.

Olivar-Julián, FJ. y Díaz-Campo, J. (2020). Jóvenes y sucesos: Tratamiento informativo en la prensa digital española. Estudios sobre el Mensaje Periodístico 26 (4), 1521-1532. doi: 10.5209/esmp.68003. 
OMS (2000). «Prevención del suicidio, un instrumento para profesionales de los medios de comunicacion», Trastornos Mentales y Cerebrales Departamento de Salud Mental y Toxicomanías. Ginebra: Organización Mundial de la Salud.

OMS (2018).Suicidio. Disponible en: https://www.who.int/es/news-room/fact-sheets/detail/suicide (Accedido: 19 de marzo de 2019).

Parks, P. (2019). From sensation to stigma: Changing standards for suicide coverage in US journalism textbooks, 18942016. Journalism, 1464884919841920.

Peña-Fernández, S., Lazkano-Arrillaga, I. y García-González, D. (2016).«European Newspapers’ Digital Transition: New Products and New Audiences», Comunicar, 24(46), pp. 27-36. doi: 10.3916/C46-2016-03.

Pérez Pereiro, M., Chaparro Dominguez, M. Á. C. y Díaz del Campo, J.(2018). La cobertura periodística de los incendios de Galicia y Portugal de octubre de 2017: un análisis de la información de emergencia de diarios portugueses, españoles y gallegos. Estudos em Comunicação, 26(1), 197-213..

Phillips, D. (1974). The influence of suggesions on suicide; substantive and theoretical implications of the Werther effect. Am. Sociol. Rev. 39: 340-354.

Pirkis, J. et al. (2019). «Suicide Prevention Media Campaigns: A Systematic Literature Review», Health Communication, 34(4), pp. 402-414. doi: 10.1080/10410236.2017.1405484.

Potvin Kent, M. y Pauzé, E. (2018). «The Frequency and Healthfulness of Food and Beverages Advertised on Adolescents' Preferred Web Sites in Canada», Journal of Adolescent Health, 63(1), pp. 102-107. doi: 10.1016/j.jadohealth.2018.01.007.

Repiso, R., Chaparro Domínguez, M. A. (2018).«Universidades españolas en la prensa extranjera. Análisis de su cobertura periodística», El profesional de la información (EPI), 27(1), pp. 86-94.

Rodríguez, A. B. (2018). «2000-2017: años dorados y oscuros para la prensa de distribución gratuita en España», Question, 1(60), p. 101. doi: http://orcid.org/0000-0002-8779-1110.

RTVE (2019).Día Internacional de la Juventud: ¿Hasta cuándo somos jóvenes? Disponible en: https://www.rtve.es/noticias/20190807/dia-internacional-juventud-hasta-cuando-somos-jovenes/1976765.shtml (Accedido: 7 de mayo de 2020).

Rubio Conesa, M. T. (2016). «Análisis de los recursos lingüísticos utilizados por los defensores del lector en pro de la confianza de los usuarios de la prensa», Revista de Investigación Lingüistica, 19.

Sindoni, M. G. (2020). «\#YouCanTalk’: A multimodal discourse analysis of suicide prevention and peer support in the Australian BeyondBlue platform», Discourse \& Communication, 14(2), pp. 202-221. doi: 10.1177/1750481319890386.

Stack, S. (2005). Suicide in the media: A quantitative review of studies based on nonfictional stories. Suicide and Life-ThreateningBehavior, 35(2), 121-133.

Till, B., Tran, U. S. y Niederkrotenthaler, T. (2020). «The Impact of Educative News Articles about Suicide Prevention: A Randomized Controlled Trial», Health Communication, pp. 1-8. doi: 10.1080/10410236.2020.1813953. 
Trilling, D., Tolochko, P. y Burscher, B. (2017). «From Newsworthiness to Shareworthiness: How to Predict News Sharing Based on Article Characteristics», Journalism and Mass Communication Quarterly, 94(1), pp. 38-60. doi: $10.1177 / 1077699016654682$.

Victor, J. et al. (2019). «Media reporting of suicide: A comparative framing analysis of Malaysian newspapers», SEARCH (Malaysia), 11(2), pp. 73-88.

Von Goethe, J. W. (1774). Die Leiden des jungen Werther. Leipzig: Insel-Verlag.

Wake, A., Paton, E. y Pryor, R. (2020). CCommunicating about suicide during a global pandemic: impact on journalists and media audiences», Media International Australia, p. 1329878X2095641. doi: 10.1177/1329878X20956415.

Yaqub, M. M., Beam, R. A. y John, S. L. (2020). “'We report the world as it is, not as we want it to be': Journalists' negotiation of professional practices and responsibilities when reporting on suicide», Journalism, 21(9), pp. 1283-1299. doi: $10.1177 / 1464884917731957$.

Zimmermann, B. M. et al. (2019). «Content, evaluations and influences in newspaper coverage of predictive genetic testing: A comparative media content analysis from the United Kingdom and Switzerland», Public Understanding of Science, 28(3), pp. 256-274. doi: 10.1177/0963662518816014. 